\title{
Integrating hearing loss was a complex and dynamic process for patients
}

\author{
Herth K. Integrating hearing loss into one's life. Qual Health Res 1998 Mar;8:207-23.
}

\section{Question}

What are the experiences of people who are integrating a hearing loss into their daily lives?

\section{Design}

Phenomenology.

\section{Setting}

A large urban area of Georgia, USA.

\section{Patients}

32 people (age range $18-84$ y, 59\% women) who reported hearing impairment defined as a loss of hearing that required the individual to develop alternate techniques for accomplishing those things that could be readily done with hearing. Hearing impairment was defined as mild, moderate, severe, or profound and was acquired after language development. All participants felt that the loss interfered with communication with others. Participants were $\geqslant 18$ years old and were recruited from churches and physicians' offices. 7 participants were 18-39 years old, 12 were 40-62 years old, and 13 were 63-84 years old. 15 were white, 10 were African-American, 4 were Asian-American, and 3 were Hispanic. 11 had completed high school. 14 were retired. 21 had an adequate financial status. 26 had had their hearing loss for 2-4 years. 27 had had a gradual onset of the hearing impairment. 5 participants had mild loss, 14 had moderate, 8 had severe, and 5 had profound hearing loss.

\section{Methods}

Semistructured interviews were done in the participant's home or designated location and included questions on what the hearing loss meant to the person, how it affected their day to day lives, what changes had been or would be made because of the hearing loss, and their beliefs of what would happen in the future. The data were transcribed and analysed and themes were developed. 6 participants were interviewed a second time to confirm the themes that emerged.

\section{Main results}

The core theme was one of dancing with. Participants felt that integrating a hearing loss into their lives was an ongoing process of adjustment. The first major theme was dancing with loss and fear. It involved loss of feeling capable, control and independence, connectedness and belonging, dignity, and self esteem plus fear of failure, dependency, ridicule, being slighted or avoided, being made conspicuous, new situations, people and chance encounters, and sudden noises.

The second theme was dancing with fluctuating feelings including grief, anger, denial, frustration, isolation, depression, loneliness, inadequacy, sadness, sorrow, discouragement, distress, and uncertainty.

The third major theme was dancing with courage amidst change. It involved changing and adding new routines, planning in advance, a shift in thinking, expenditure of energy, and engendering courage and resilence.

The fourth theme was dancing with an altered life perspective. It involved seeking support, gaining information, developing new problem solving skills, implementing new approaches, using humour, and finding hope and meaning in their hearing loss.

\section{Conclusion}

Integrating a hearing loss was a complex and dynamic process and often involved a long and lonely struggle to deal with the limitations on communication with others and the outside world.

Source of funding: no external funding.

For correspondence: Dr K Herth, Associate Dean, School of Nursing, Box 27, Mankato State University, PO Box 8400, Mankato, MN 56002-8400, USA. Fax +1 5073896516.

\section{Commentary}

The study by Herth uses phenomenological research to explore the effect of hearing loss in adults. Hearing loss is one of the most common chronic physical disabilities; its prevalence and severity increase dramatically with age. Presbycusis (alteration in hearing capacity related to aging) affects $13 \%$ of people $>65$ years old. ${ }^{1} 30 \%$ of people $>65$ years have hearing loss that requires a hearing aid. Nurses have reason to learn more about hearing loss-the aetiology, incidence, and social isolation of the quiet world-as we care for an aging population. An understanding of how people integrate hearing loss into their lives is fundamental to the design and evaluation of assessment and intervention strategies related to this chronic disability.

The study participants had a range of ages and aetiologies for hearing loss, both abrupt and gradual in onset. The study sought to provide insight about how these 32 people perceived their situation and did not set out to make statements about the population of hearing impaired adults in the US. Nurses should be cautious about the general applicability of the 4 study themes beyond this sample. The analysis was done by 1 researcher and theme saturation was confirmed by a second interview with only 6 members of the original sample.

A strength of this study was the liberal inclusion of anecdotal material to substantiate the theme descriptions. Nurses who read the original article will be able to extrapolate from some of this anecdotal material in 3 ways. Firstly, they may modulate their own voice tone to clear slow speech at a low pitch when assessing hearing ability in client populations in the community or in institutions. Secondly, they may investigate the resources in their community to shift to visual cues for clients who have difficulty hearing the doorbell, telephone, or oven timer. Thirdly, nurses can now critique descriptions of environmental modifications such as the New England Home for the Deaf with additional sensitivity and intention to address the social isolation associated with hearing loss. ${ }^{2}$

Replication of this study design using other populations with sensory or communication loss is also a consideration for further research. The richness of qualitative data complements many research modalities which may be selected in clinical practice.

Beverly L Jones, RN, MScN Clinical Nurse Specialist Kingston Psychiatric Hospital Kingston, Ontario, Canada

1 Wold G. Basic geriatric nursing. St Louis: CV Mosby, 1993.

2 Greene AW, Mosher-Ashley PM. A residential care alternative for elderly deaf persons. J Gerontol Nurs 1997;23:32-6. 